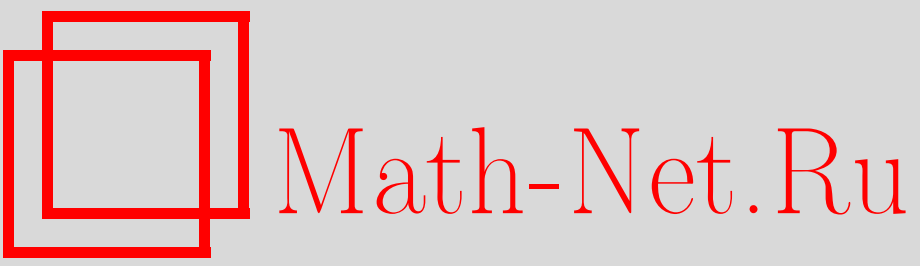

Ф. Г. Рагимов, Об асимптотическом поведении локальных вероятностей пересечения нелинейных границ многомерным случайным блужданием, Теория вероятн. и ее примен., 2009, том 54, выпуск 2, 367-374

DOI: https://doi.org/10.4213/tvp2712

Использование Общероссийского математического портала Math-Net.Ru подразумевает, что вы прочитали и согласны с пользовательским соглашением

http://www . mathnet.ru/rus/agreement

Параметры загрузки:

IP: 54.89 .56 .158

26 апреля 2023 г., 16:25:37 
Но из $(16)$ следует, что $\gamma(U)>\gamma(T)$, т.е. тесты, основанные на $U_{n}$, при альтернативах $H_{1}$ в пределе более мощны, чем тесты, основанные на $T_{n}$. Более того, интегрируя $f_{1}(x)$, можно установить, что альтернативы отличаются от нулевой гипотезы на величину порядка $\alpha_{n} \gamma_{n}=o(1 / \sqrt{n})$. Стало быть, мы имеем более высокую предельную мощность против такой альтернативы в случае критериев, основанных на $U_{n}$ и $T_{n}$, чем в случае критериев, основанных на отклонении эмпирической функции распределения от истинной, например критерия $\omega_{n}^{2}$ и критерия Колмогорова-Смирнова.

\section{СПИСОК ЛИТЕРАТУРЫ}

1. Надарая Э. А. О предельном распределении квадратического уклонения обобщенной ядерной оценки плотности распределения. - Теория вероятн. и ее примен., 1992, т. 37, в. 2 , с. $413-421$.

2. Липцер P.Ш., Ширяев А.Н. Функциональная центральная предельная теорема для семимартингалов. - Теория вероятн. и ее примен., 1980 , т. 25 , в. 4, с. $683-$ 703.

3. Nadaraya E., Babilua $P$. On the limit distribution of a quadratic deviation of a recurrent kernel estimate of distribution density. - Proc. A. Razmadze Math. Inst., 2007, v. 144, p. $67-89$.

4. Nadaraya E., Babilua P. On the Wolverton-Wagner estimate of a distribution density. - Bull. Georgian National Acad. Sci., 2007, v. 175, № 1, p. 40-44.

5. Крамер Г. Математические методы статистики. М.: Мир, 1975, 648 с.

6. Bickel P. J., Rosenblatt M. On some global measures of the deviations of density function estimates. - Ann. Statist., 1973, v. 1, p. 1071-1095.

7. Rosenblatt $M$. A quadratic measure of deviation of two-dimensional density estimates and a test of independence. - Ann. Statist., 1975, v. 3, p. 1-14.

8. Надарая Э. А. Непараметрическое оценивание плотности вероятностей и кривой регрессии. Тбилиси, 1983, 194 с.

9. Ghosh B.K., Huang W.-M. The power and optimal kernel of the Bickel-Rosenblatt test for goodness of fit. - Ann. Statist., 1991, v. 19, № 2, p. 999-1009.

Поступила в редакцию

17.II.2009

(c) 2009 г.

РАГИМОВ Ф. Г.*

\section{ОБ АСИМПТОТИЧЕСКОМ ПОВЕДЕНИИ ЛОКАЛЬНЫХ ВЕРОЯТНОСТЕЙ ПЕРЕСЕЧЕНИЯ НЕЛИНЕЙНЫХ ГРАНИЦ МНОГОМЕРНЫМ СЛУЧАЙНЫМ БЛУЖДАНИЕМ}

В настоящей работе доказана теорема об асимптотическом поведении совместного распределения перескока и момента первого выхода для случайного блуждания, описываемого нелинейной функцией от сумм независимых случайных векторов. В качестве следствия получены локальная предельная теорема и асимптотическая независимость перескока (эксцесса) и момента первого выхода.

Ключевые слова и фразы: случайное блуждание, момент первого выхода, перескок (эксцесс), локальная предельная теорема.

* Бакинский государственный университет, факультет прикладной математики и кибернетики, кафедра теории вероятностей и математической статистики, ул. З. Халилова, 23, Az 1148, Баку, Республика Азербайджан; е-mail: ragimovf@rambler.ru 
1. Введение. Пусть $\xi_{n}, n \geqslant 1$, - последовательность независимых одинаково распределенных случайных векторов в $\mathbf{R}^{k}, k \geqslant 1$, со средним значением $\nu=\mathbf{E} \xi_{1}$, и пусть $\Delta(X), x \in \mathbf{R}^{k}$, - некоторая числовая борелевская функция.

Положим при $n \geqslant 1$ и $c \geqslant 0$

$$
S_{n}=\sum_{i=1}^{n} \xi_{i}, \quad T_{n}=n \Delta\left(\frac{S_{n}}{n}\right)
$$

и рассмотрим момент первого выхода

$$
\tau=\tau_{c}=\inf \left\{n \geqslant 1: T_{n}>c\right\}
$$

процесса $T_{n}$ за уровень $c \geqslant 0$. Будем считать, что $\inf \{\varnothing\}=\infty$.

Многие важные моменты остановки, возникающие в статистическом последовательном анализе и в теории нелинейного восстановления, имеют вид (1) (см., например, [1], [2]).

В работе [5] изучены интегральные предельные теоремы для $\tau_{c}$, из которых, в частности, следует, что для фиксированных чисел $a \geqslant 1$ и $b \geqslant 1$ вероятность $\mathbf{P}\left(a \leqslant \tau_{c}<b\right)$ стремится к нулю при $c \rightarrow \infty$. При оценке вероятности событий вида $\left\{\tau_{c} \in(a, b)\right\}$ существенную роль играют локальные предельные теоремы, под которыми понимаются любые утверждения о том, что при некоторых условиях существует функция $P(n, c)$, для которой выполняется следующее соотношение:

$$
\mathbf{P}\left(\tau_{c}=n\right)=P(n, c)(1+o(1)) \quad \text { при } c \rightarrow \infty .
$$

В 1970-90-х годах был достигнут существенный прогресс в области локальных предельных теорем для момента первого выхода одномерного случайного блуждания (cм. [1], [3], [4]).

Этому прогрессу в значительной степени способствовали метод факторизационных тождеств при $\Delta(x)=x$ (см. [4]) и так называемый локальный метод Вудруфа при $\Delta(x) \neq x($ см. $[1]-[3])$.

Многомерный случай (т.е. случай $k>1$ ) изучен существенно меньше. Ряд общих результатов в этом направлении имеется в работах [6] и [7]. Как отмечалось в [7], это связано прежде всего с тем, что аналитические методы, сыгравшие важную роль в одномерном случае, для многомерных блужданий являются неэффективными. Тем не менее, ряд граничных задач, связанных с моментом первого выхода вида (1), удается решить (см. [2], [5]). В [2] изучаются некоторые задачи последовательного анализа, в которых возникают моменты остановки $\tau_{c}$ вида (1) с $\Delta(x)=\|x\|^{2}$, где $\|\cdot\|$ - обычная евклидова норма в $\mathbf{R}^{k}$.

Целью настоящей работы является изучение асимптотического поведения совместного распределения момента первого выхода $\tau_{c}$ и перескока $\chi_{c}=T_{c}-c$, а также вероятности $\mathbf{P}\left(\tau_{c}=n\right)$ для достаточно широкого класса функций $\Delta(x)$ и случайных блужданий.

2. Условия и обозначения. Пусть $a=\left(a_{1}, \ldots, a_{k}\right) \in \mathbf{R}^{k}$ и $b=\left(b_{1}, \ldots, b_{k}\right) \in \mathbf{R}^{k}$. Будем писать $a \leqslant b$, если $a_{i} \leqslant b_{i}$ для каждого $i=1, \ldots, k$. Положим

$$
D=D(d, y)=\bigcup_{m=-\infty}^{\infty}\left\{x \in \mathbf{R}^{k}:(d, x-y)=m\right\} \text {. }
$$

Если существуют векторы $y \in \mathbf{R}^{k}$ и $d \in \mathbf{R}^{k}$ такие, что $\mathbf{P}\left(\xi_{1} \in D\right)=1$, то говорят, что $\xi_{1}$ имеет решетчатое распределение с параметром $d \in \mathbf{R}^{k}$. В противном случае распределение случайного вектора $\xi_{1}$ будем называть нерешетчатым.

Обозначим через $W_{\nu}$ класс функций $\Delta(x), x \in \mathbf{R}^{k}$, для которых выполняются следующие условия: положительная функция $\Delta(x)$ имеет непрерывные частные производные $\Delta_{x_{i}}^{\prime}(x), i=1, \ldots, k$, в некоторой окрестности точки $x=\nu \in \mathbf{R}^{k}$, причем 
$\Delta(\nu)>0$ и $\Delta_{x_{i}}^{\prime}(\nu) \neq 0$ хотя бы для одного $i=1, \ldots, k$. Ниже $(x, y)$ означает обычное скалярное произведение векторов $x, y \in \mathbf{R}^{k}$.

Отметим, что при сделанных предположениях относительно функции $\Delta(x)$ имеем:

$$
T_{n}=Z_{n}+\varepsilon_{n},
$$

где

$$
\begin{aligned}
& Z_{n}=\sum_{i=1}^{n} X_{i}, \quad X_{i}=\Delta(\nu)+\left(\lambda, \xi_{i}-\nu\right), \\
& \varepsilon_{n}=n\left(\frac{1}{n} S_{n}-\nu, \lambda_{n}-\lambda\right), \quad \begin{aligned}
\lambda_{n} & =\left(\Delta_{x_{1}}^{\prime}\left(\nu_{n}\right), \ldots, \Delta_{x_{k}}^{\prime}\left(\nu_{n}\right)\right), \\
\lambda & =\left(\Delta_{x_{1}}^{\prime}(\nu), \ldots, \Delta_{x_{k}}^{\prime}(\nu)\right),
\end{aligned}
\end{aligned}
$$

и $\nu_{n}, n \geqslant 1$, - некоторая последовательность случайных точек из окрестности $\nu=\mathbf{E} \xi_{1}$, причем $\nu_{n} \rightarrow \nu$ п.н. при $n \rightarrow \infty$. Нетрудно понять, что последовательность $Z_{n}, n \geqslant 1$, есть одномерное случайное блуждание с $\mathbf{E} Z_{1}=\Delta(\nu)>0$.

Обозначим

$$
H(x)=\frac{1}{\mathbf{E} Z_{\tau_{+}}} \int_{0}^{x} \mathbf{P}\left(Z_{\tau_{+}}>y\right) d y,
$$

где

$$
Z_{n}=n \Delta(\nu)+\left(\lambda, S_{n}-n \nu\right)
$$

и $\tau_{+}=\inf \left\{n \geqslant 1: Z_{n}>0\right\}$.

\section{3. Формулировка и доказательство основных результатов.}

Теорема 1. Пусть $\xi_{n}, n \geqslant 1$, - последовательность независимых одинаково распределенных нерешетчатых случайных векторов с вектором математических ожиданий $\nu=\mathbf{E} \xi_{1}$ и матрищей ковариачий $B$, и пусть $\Delta(x) \in W_{\nu}$. Если

$$
n=n(c)=\frac{c}{\Delta(\nu)}+\theta(c) \sqrt{\frac{c}{\Delta(\nu)}},
$$

причем $\theta(c) \rightarrow \theta \in \mathbf{R}$ при $c \rightarrow \infty$, mo

$$
\mathbf{P}\left(\tau_{c}=n, \chi_{c} \leqslant x\right) \sim \frac{\Delta(\nu)}{\sqrt{n}} \varphi_{\gamma}(-\theta \Delta(\nu)) H(x)
$$

равномерно по $x, 0<\delta \leqslant x \leqslant M<\infty, u \theta$ из ограниченного множества в $\mathbf{R}$, где $\varphi_{\gamma}(x)$ - плотность нормального распределения с параметрами $(0, \gamma), \gamma=\lambda B \lambda^{\mathrm{T}}$ $\left(\lambda^{\mathrm{T}}-\right.$ вектор-столбец, транспонированный $\left.\kappa \lambda\right)$.

Следствие 1. Если выполнень условия теоремь 1, то

$$
\mathbf{P}\left(\tau_{c}=n\right) \sim \frac{\Delta(\nu)}{\sqrt{n}} \varphi_{\gamma}(-\theta \Delta(\nu)) \quad \text { npu } c \rightarrow \infty .
$$

Отметим, что теорема 1 допускает следующее расширение для случая, когда распределение случайного вектора $\xi_{1}$ принадлежит области притяжения многомерного устойчивого распределения в смысле Леви-Фельдхейма с характеристическим показателем $\alpha \in(1,2]$ (см. [9], [10]).

Теорема 2. Пусть $\xi_{n}, n \geqslant 1$, - последовательность независимьх одинаково распределенных случайных векторов в $\mathbf{R}^{k}$ с конечным вектором математических ожиданий $\nu=\mathbf{E} \xi_{1} u \Delta(x) \in H_{\nu}$. Предположим, ито существуют медленно меняющаяся на бесконечности функиия $L(x), x>0, u k$-мерный случайнылй вектор $J$ такие, чmо

$$
\frac{S_{n}-n \nu}{A(n)} \Rightarrow J \quad \text { nрu } n \rightarrow \infty, \quad \text { где } A(n)=n^{1 / \alpha} L(n), \quad \alpha \in(1,2] .
$$


Eсли $n=n(c)=c / \Delta(\nu)+\theta(c) A([c / \Delta(\nu)])$, причем $\theta(c) \rightarrow \theta \in \mathbf{R}$ при $c \rightarrow \infty$, mo

$$
\mathbf{P}\left(\tau_{c}=n, \chi_{c} \leqslant x\right) \sim \frac{\Delta(\nu)}{A(n)} g_{a}(-\theta \Delta(\nu)) H(x)
$$

равномерно по $x, 0<\delta \leqslant x \leqslant M<\infty$, и $\theta$ из ограниченного множества в $\mathbf{R}$, где $g_{a}(x)$ - плотность устойчивого распределения случайной величинь $(\lambda, J)$.

Следствие 2. Пусть выполняются условия теоремы 2. Тогда

$$
\mathbf{P}\left(\tau_{c}=n\right) \sim \frac{\Delta(\nu)}{A(n)} g_{a}(-\theta \Delta(\nu)) \quad \text { nрu } c \rightarrow \infty .
$$

Следствие 3. При выполнении условий теоремь 1 (или теоремы 2) имеет меcmo

$$
\lim _{c \rightarrow \infty} \mathbf{P}\left(\chi_{c} \leqslant x \mid \tau_{c}=n\right)=H(x), \quad x>0 .
$$

Этот результат показывает, что момент первого выхода $\tau_{c}$ и перескок $\chi_{c}$ асимптотически независимы (см. [1], [4]).

Отметим, что в условиях теоремы 2 имеет место сходимость по распределению:

$$
\frac{Z_{n}-n \Delta(\nu)}{A(n)} \Rightarrow(\lambda, J) \text {. }
$$

Это означает, что распределение случайной величины $X_{1}=\Delta(\nu)+\left(\lambda, \xi_{1}-\nu\right)$ принадлежит области притяжения устойчивого распределения случайной величины $(\lambda, J)$ с характеристическим показателем $\alpha \in(1,2]$ (см. [9]).

Для доказательства теоремы 1 нам понадобится ряд утверждений, сформулированных в виде следующих лемм.

Лемма 1. Пусть $\eta_{n}, n \geqslant 1$, - независимые одинаково распределенные случайные величины с $\sigma^{2}=\mathbf{D} \eta_{1} и a=\mathbf{E} \eta_{1}$. Для того чтобы для любого ограниченного выпуклого борелевского множества $B$ в $\mathbf{R}$ равномерно по $x \in \mathbf{R}$ выполнялось соотношение

$$
\mathbf{P}\left(\sum_{i=1}^{n} \eta_{i} \in x+B\right)=\frac{\mu(B)}{\sigma \sqrt{n}} \varphi\left(\frac{x-n a}{\sigma \sqrt{n}}\right)+o\left(\frac{1}{\sqrt{n}}\right),
$$

необходимо и достаточно, чтобы распределение случайной величины $\eta_{1}$ было нере-

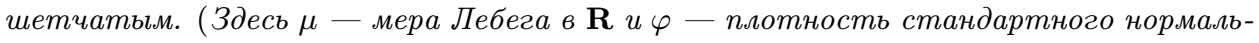
ного распределения.)

Этот результат содержится в качестве следствия основного результата в [8].

Лемма 2. Пусть случайный вектор $\xi_{1}$ имеет нерешетчатое распределение и $\Delta(x) \in H_{\nu}$. Тогда

$$
\lim _{c \rightarrow \infty} \mathbf{P}\left(\chi_{c} \leqslant x\right)=H(x), \quad x \geqslant 0 .
$$

Утверждение этой леммы является частным случаем теорем 2.7 и 4.1 из работь [1] в силу разложения $T_{n}=Z_{n}+\varepsilon_{n}$.

Лемма 3. Предельное распределение $H(x)$ имеет плотность

$$
h(x)=\frac{1}{\Delta(\nu)} \mathbf{P}\left(Z_{i} \geqslant x, i \geqslant 1\right) .
$$

Эта лемма следует из теорем 2.7 и 4.1 работы [1] в силу разложения $T_{n}=Z_{n}+\varepsilon_{n}$.

Док а з а те ль с т в о т е о р е м ы 1 . Разделим промежуток $(0, x]$ на $m$ равных частей и положим $l_{j}=((j-1) x / m, j x / m], j=1, \ldots, m$. По формуле полной вероятности имеем:

$$
\begin{aligned}
\mathbf{P}\left(\tau_{c}=n, \chi_{c} \leqslant x\right) & =\sum_{j=1}^{m} \mathbf{P}\left(\tau_{c}=n, T_{n} \in c+l_{j}\right) \\
& =\sum_{j=1}^{m} \mathbf{P}\left(\tau_{c}=n \mid T_{n} \in c+l_{j}\right) \mathbf{P}\left(T_{n} \in c+l_{j}\right) .
\end{aligned}
$$


Оценим условную вероятность $Q_{n, j}(c)=\mathbf{P}\left(\tau_{c}=n \mid T_{n} \in c+l_{j}\right)$. Нетрудно видеть, что

$$
\begin{aligned}
Q_{n, j}(c) & =\mathbf{P}\left(\tau_{c} \geqslant n \mid T_{n} \in c+l_{j}\right)=\mathbf{P}\left(T_{i} \leqslant c, 1 \leqslant i<n \mid T_{n} \in c+l_{j}\right) \\
& =\mathbf{P}\left(T_{n}-T_{i} \geqslant T_{n}-c, 1 \leqslant i<n \mid T_{n} \in c+l_{j}\right) \\
& =\mathbf{P}\left(T_{n}-T_{n-i} \geqslant T_{n}-c, 1 \leqslant i<n \mid T_{n} \in c+l_{j}\right) .
\end{aligned}
$$

Отсюда имеем

$$
\begin{aligned}
\mathbf{P}\left(T_{n}-T_{n-i}\right. & \left.\geqslant \frac{j}{m} x, 1 \leqslant i<n \mid T_{n} \in c+l_{j}\right) \\
& \leqslant Q_{n, j}(c) \leqslant \mathbf{P}\left(T_{n}-T_{n-i} \geqslant \frac{j-1}{m} x, 1 \leqslant i<n \mid T_{n} \in c+l_{j}\right) .
\end{aligned}
$$

Обозначим $\Pi_{n, j}(x, c)=\mathbf{P}\left(T_{n}-T_{n-i} \geqslant j x / m, 1 \leqslant i<n \mid T_{n} \in c+l_{j}\right)$. Из (2) получаем, что

$$
\begin{aligned}
\sum_{j=1}^{m} \Pi_{n, j}(x, c) \mathbf{P}\left(T_{n} \in c+l_{j}\right) & \leqslant \mathbf{P}\left(\tau_{c}=n, \chi_{c} \leqslant x\right) \\
& \leqslant \sum_{j=1}^{m} \Pi_{n, j-1}(x, c) \mathbf{P}\left(T_{n} \in c+l_{j}\right) .
\end{aligned}
$$

Далее нам потребуются следующие леммы.

Лемма 4. Для любого $\varepsilon>0$ существует челое $q_{1}$ такое, что для достаточно больших с и для всех $r$ и х из ограниченного множества выполнено неравенство

$$
\max _{j \leqslant m} \mathbf{P}\left(J_{n i} \leqslant r, \exists i \in\left[q_{1}, n\right) \mid T_{n} \in c+l_{j}\right)<\varepsilon, \quad \text { где } J_{n i}=T_{n}-T_{n-i} .
$$

Оценка (4) для случая $k=1$ доказана в работе [4]. В общем случае ее можно доказать с помощью выкладок и рассуждений, аналогичных проведенным в работе [4].

Лемма 5. Для каждого фиксированного челого числа $p \geqslant 1$ совместное условное распределение случайных величин

$$
J_{n i}=T_{n}-T_{n-i}, \quad i=1, \ldots, p,
$$

при условии $n^{-1} S_{n}=y\left(y \in \mathbf{R}^{k}\right.$ фиксировано) слабо сходится $\kappa$ безусловному распределению случайных величин $Z_{1}, \ldots, Z_{p} u$

$$
\lim _{n \rightarrow \infty} \mathbf{P}\left(J_{n i} \geqslant x, 1 \leqslant i \leqslant p \mid T_{n} \in c+l_{j}\right)=\mathbf{P}\left(Z_{i} \geqslant x, 1 \leqslant i \leqslant p\right) .
$$

Утверждения этой леммы для одномерного случая содержатся в [1]. В многомерном случае их можно показать с помощью рассуждений, проведенных при доказательстве теоремы 5.1 из [1] (см. также [4]).

Продолжим доказательство теоремы 1. Поскольку $\mathbf{E} Z_{1}=\Delta(\nu)>0$, то из усиленного закона больших чисел вытекает, что для любого $\varepsilon>0$ существует достаточно большое целое число $q_{2}$ такое, что

$$
\mathbf{P}\left(Z_{j} \leqslant x, \exists j>q_{2}\right)<\varepsilon .
$$

Полагая $q=\max \left(q_{1}, q_{2}\right)$ вместо $q_{1}$ в (4) и вместо $q_{2}$ в (6), имеем

$$
\begin{aligned}
\mathbf{P}\left(J_{n i} \geqslant x, 1 \leqslant i \leqslant q \mid T_{n} \in c+l_{j}\right)-\varepsilon & \leqslant \mathbf{P}\left(J_{n i} \geqslant x, 1 \leqslant i<n \mid T_{n} \in c+l_{j}\right) \\
& \leqslant \mathbf{P}\left(J_{n i} \geqslant x, 1 \leqslant i \leqslant q \mid T_{n} \in c+l_{j}\right)
\end{aligned}
$$

и

$$
\mathbf{P}\left(Z_{i} \geqslant x, 1 \leqslant i \leqslant q\right)-\varepsilon \leqslant \mathbf{P}\left(Z_{i} \geqslant x, 1 \leqslant i\right) \leqslant \mathbf{P}\left(Z_{i} \geqslant x, 1 \leqslant i \leqslant q\right) .
$$

Из соотношения (5) при $p=q$ и неравенств (7) и (8) получаем, что

$$
\begin{aligned}
\mathbf{P}\left(Z_{i} \geqslant x, i \geqslant 1\right)-2 \varepsilon & \leqslant \mathbf{P}\left(J_{n i} \geqslant x, 1 \leqslant i<n \mid T_{n} \in c+l_{j}\right) \\
& \leqslant \mathbf{P}\left(Z_{i} \geqslant x, i \geqslant 1\right)+2 \varepsilon .
\end{aligned}
$$


Тогда из (9) и (3) имеем:

$$
\begin{gathered}
\sum_{j=1}^{m} \frac{x}{m} \mathbf{P}\left(Z_{i} \geqslant \frac{j}{m} x, i \geqslant 1\right) \mathbf{P}\left(T_{n} \in c+l_{j}\right)-2 \varepsilon \leqslant \mathbf{P}\left(\tau_{c}=n, \chi_{c} \leqslant x\right) \\
\leqslant \sum_{j=1}^{m} \mathbf{P}\left(Z_{i} \geqslant \frac{j-1}{m} x, i \geqslant 1\right) \mathbf{P}\left(T_{n} \in c+l_{j}\right)+2 \varepsilon
\end{gathered}
$$

Теперь оценим вероятности $\mathbf{P}\left(T_{n} \in c+l_{j}\right)$. В силу леммы 1

$$
\mathbf{P}\left(Z_{n} \in c+l_{j}\right)=\frac{\mu\left(J_{j}\right)}{\sqrt{n}} \varphi_{\gamma}\left(\frac{c-n \Delta(\nu)}{\sqrt{n}}\right)+o\left(\frac{1}{\sqrt{n}}\right)
$$

или

$$
\mathbf{P}\left(Z_{n} \in c+l_{j}\right) \sim \frac{x / m}{\sqrt{n}} \varphi_{\gamma}(-\theta \Delta(\nu))
$$

Учитывая, что $T_{n}=Z_{n}+\varepsilon_{n}$ и $\varepsilon_{n} / \sqrt{n} \stackrel{\mathbf{P}}{\rightarrow} 0$ при $n \rightarrow \infty$, из (11) находим, что

$$
\mathbf{P}\left(T_{n} \in c+l_{j}\right) \sim \frac{x / m}{\sqrt{n}} \varphi_{\gamma}(-\theta \Delta(\nu))
$$

Подставляя (12) в (10), имеем:

$$
\begin{gathered}
\varphi_{\gamma}(-\theta \Delta(\nu))(1-\varepsilon) \sum_{j=1}^{m} \frac{x}{m} \mathbf{P}\left(Z_{i} \geqslant \frac{j}{m} x, i \geqslant 1\right)-2 \varepsilon \leqslant \sqrt{n} \mathbf{P}\left(\tau_{c}=n, \chi_{c} \leqslant x\right) \\
\leqslant(1+\varepsilon) \varphi_{\gamma}(-\theta \Delta(\nu)) \sum_{j=1}^{m} \frac{x}{m} \mathbf{P}\left(Z_{i} \geqslant \frac{j-1}{m} x, i \geqslant 1\right)+2 \varepsilon .
\end{gathered}
$$

Согласно лемме 3 ,

$$
\mathbf{P}\left(Z_{i} \geqslant x, i \geqslant 1\right)=\frac{\Delta(\nu)}{\mathbf{E} Z_{\tau_{+}}} \mathbf{P}\left(Z_{\tau_{+}}>x\right)
$$

Поэтому из (13), выбирая сначала $\varepsilon$ сколь угодно малым, а затем $m$ достаточно большим, находим, что

$$
\mathbf{P}\left(\tau_{c}=n, \chi_{c} \leqslant x\right)=\frac{\Delta(\nu)}{\sqrt{n}} \varphi_{\gamma}(-\theta \Delta(\nu)) H(x)+o\left(\frac{1}{\sqrt{n}}\right)
$$

равномерно по $x$ из ограниченного множества в $[\delta, \infty)$ с $\delta>0$ и по $\theta$ из ограниченного множества в $\mathbf{R}$.

Теорема 1 доказана.

Доказательство теоремы 2 проводится по схеме доказательства теоремы 1 , при этом ключевую роль играет следующая лемма.

Лемма 6. Пусть выполняются условия теоремь 2. Тогда при с $\rightarrow \infty$

$$
\mathbf{P}\left(\sum_{i=1}^{n} X_{i} \in x+B\right) \sim \frac{h}{A(n)} g_{a}(-\theta \Delta(\nu))
$$

для любого ограниченного выпуклого борелевского множества в $B$ с $\mu(B)=h>0$, где $\mu$ - мера Лебега в $\mathbf{R}$.

Д о к а з а т е л с т в о. При выполнении условий теоремы 2 имеет место основная теорема работы [8], согласно которой

$$
\mathbf{P}\left(\sum_{i=1}^{n} X_{i} \in x+B\right)=\frac{h}{A(n)} g_{a}\left(\frac{x-n \Delta(\nu)}{A(n)}\right)+o\left(\frac{1}{A(n)}\right) .
$$


Подставляя в эту формулу разложение для $n=n(c)$ из условий теоремы 2 , получаем утверждение леммы 6.

Следствия 1 и 2 вытекают соответственно из теорем 1 и 2 при $x \rightarrow \infty$. Следствие 3 вытекает из следствия 2 и теоремы 2.

3 а м е ч а н и е. Из хода доказательства теоремы 1 следует, что ее утверждение имеет место равномерно по $x$ из конечного интервала в $[\delta, \infty)$ с $\delta>0$. Для выполнения утверждения этой теоремы равномерно по $x \in[\delta, \infty)$ требуется дополнительное условие на функцию $\Delta(x)$ и на распределение случайной величины $\Delta\left(\xi_{1}\right)$. Например, если $\Delta(x)$ является выпуклой в некоторой окрестности точки $x=\nu$, то условие

$$
\mathbf{E}\left(\Delta\left(\xi_{1}\right)\right)^{+}<\infty
$$

обеспечивает выполнение утверждение теоремы 2 для $x \in[\delta, \infty), \delta>0$. Действительно, из условия (14) вытекает, что для любого $\varepsilon>0$ существует достаточное большое число $x$ такое, что

$$
\sum_{l=0}^{\infty} \mathbf{P}\left(\Delta\left(\xi_{1}\right)>x+l\right)<\varepsilon .
$$

Из выпуклости функции $\Delta(x)$ в окрестности точки $x=\nu$ следует, что

$$
T_{n}=n \Delta\left(\frac{S_{n}}{n}\right)=n \Delta\left(\frac{n-1}{n} \frac{S_{n-1}}{n-1}+\frac{\xi_{n}}{n}\right) \leqslant T_{n-1}+\Delta\left(\xi_{n}\right)
$$

для достаточно больших $n$.

Следовательно,

$$
\begin{aligned}
\mathbf{P}\left(\tau_{c}=n, \chi_{c}>x\right) & =\mathbf{P}\left(\tau_{c}=n, T_{n}>c+x\right) \leqslant \mathbf{P}\left(\tau_{c}=n, \Delta\left(\xi_{1}\right)>c+x-T_{n-1}\right) \\
& \leqslant \int_{0}^{\infty} \mathbf{P}\left(\Delta\left(\xi_{1}\right)>x+z\right) \mathbf{P}\left(T_{n-1} \in c-d z\right) \\
& \leqslant \sum_{l=0}^{\infty} \mathbf{P}\left(\Delta\left(\xi_{1}\right)>x+l\right) \mathbf{P}\left(T_{n-1} \in[c-l-1, c-l]\right) .
\end{aligned}
$$

Далее, согласно лемме 1 , существует константа $M>0$ такая, что для всех $c \geqslant 0$ и $l \geqslant 0$

$$
\mathbf{P}\left(T_{n-1} \in[c-l-1, c-l]\right) \leqslant \frac{M}{\sqrt{n}} .
$$

Поэтому, учитывая (15), получаем, что $\mathbf{P}\left(\tau_{c}=n, \chi_{c}>x\right) \leqslant \varepsilon M / \sqrt{n}$. Отсюда следует, что утверждение теоремы 2 выполняется равномерно по $x$ на «крае», т.е. вблизи бесконечности.

Отметим, что это замечание остается в силе и для теоремы 2.

\section{СПИСОК ЛИТЕРАТУРЫ}

1. Woodroofe M. Nonlinear Renewal Theory in Sequential Analysis. Philadelphia: SIAM, 1982, $119 \mathrm{p}$.

2. Siegmund D. Sequential Analysis. Tests and Confidence Intervals. New York: SpringerVerlag, 1985, $272 \mathrm{p}$.

3. Lalley $S$. Limit theorems for first-passage times in linear and nonlinear renewal theory. - Adv. Appl. Probab., 1984, v. 16, № 4, p. 766-803.

4. Рагимов Ф. Г. Предельные теоремы для моментов первого выхода процессов с независимыми приращениями. Дисс. канд. физ.-матем. наук. М.: Математический институт им. В. А. Стеклова РАН, 1985, 127 с.

5. Рагимов Ф.Г. Об асимптотическом поведении одного класса моментов первого выхода за границу в многомерном случайном блуждании. - Теория вероятн. и ее. примен., 2005 , т. 50 , в. 3, с. $579-585$. 
6. Aras G., Woodroofe M. Asymptotic expansions for the moments of a randomly stopped average. - Ann. Statist., 1993, v. 21, № 1, p. 503-519.

7. Боровков A. A., Могульский A. А. Предельные теоремы в задаче достижения границы многомерным блужданием. - Сиб. матем. журн., 2001, т. 42, № 2, с. 289 317.

8. Мухин А.Б. Локальные предельные теоремы для распределений сумм независимых случайных векторов. - Теория вероятн. и ее. примен., 1984, т. 29, № 2, c. $360-366$.

9. Золотарев В. М. Одномерные устойчивые распределения. М: Наука, 1983, 304 с.

10. Рвачева E.Л. Об областях притяжения многомерных устойчивых распределений. - Ученые записки Львовского ун-та, сер. мех.-матем., 1954, т. 29, № 1, c. 5-44.

Поступила в редакцию 30.III. 2007

Исправленный вариант

11.III. 2008

(c) 2009 г

СИДОРОВ Д. И.*

\title{
ОБ УСЛОВИЯХ ПЕРЕМЕШИВАНИЯ ПОСЛЕДОВАТЕЛЬНОСТЕЙ СКОЛЬЗЯЩИХ СРЕДНИХ ${ }^{1)}$
}

\begin{abstract}
В статье изучается применимость классических условий перемешивания для последовательностей скользящих средних, построенных как по независимым, так и по зависимым случайным величинам. В частности, описан класс скользящих средних без условия $m$-зависимости, но со свойством равномерно сильного перемешивания. Также доказано отсутствие перемешивания для некоторых классов скользящих средних.
\end{abstract}

Ключевые слова и фразы: скользящие средние, сильное перемешивание, равномерно сильное перемешивание.

Введение. Пусть $\left\{\xi_{j} j \in \mathbf{Z}\right\}$ - последовательность случайных величин, заданных на одном вероятностном пространстве, $\left\{a_{j} ; j \in \mathbf{Z}\right\}$ - некоторые вещественные числа ( $\mathbf{Z}$ - множество всех целых чисел). Скользящие средние $\left\{X_{k} ; k \in \mathbf{Z}\right\}$, порожденные последовательностью $\left\{\xi_{j}\right\}$, определяются равенством

$$
X_{k}:=\sum_{j \in \mathbf{Z}} a_{k-j} \xi_{j}
$$

при этом предполагается, что ряд в (1) сходится с вероятностью 1. В частности, это будет выполнено, если

$$
\sum_{j}\left|a_{k-j}\right| \mathbf{E}\left|\xi_{j}\right|<\infty .
$$

Если же случайные величины $\left\{\xi_{j} ; j \in \mathbf{Z}\right\}$ независимы, имеют конечный второй момент и центрированы, то для сходимости ряда в (1) достаточно потребовать, чтобы выполнялось более слабое условие

$$
\sum_{j} a_{k-j}^{2} \mathbf{E} \xi_{j}^{2}<\infty
$$

* Институт математики им. С. Л. Соболева СО РАН, пр. Академика Коптюга, 4, Новосибирск 630090, Россия; e-mail: dsidorov@mail.ru

1) Работа выполнена при поддержке РФФИ (грант № 06-01-00738). 